\title{
Widening the spectrum of LAMA 2 congenital muscular dystrophy (MDC1A): cobblestone malformation
}

\author{
Ampliação do espectro da distrofia muscular congênita associada ao LAMA 2 (MDC1A): \\ malformação do tipo "cobblestone" \\ Luiz Fernando Monte BORELLA', Fernanda Veloso PEREIRA', Paula Maria Preto MIMURA², \\ Juliana Ávila DUARTE ${ }^{3}$, Luciano de Lima VILLARINHO4, Thiago REZENDE5, \\ Marcondes Cavalcante de FRANCAA JUNIOR ${ }^{5}$, Fabiano REIS ${ }^{1}$
}

A 4-year-old boy with LAMA2-related congenital muscular dystrophy had two pathogenic variants (NM_000426): c.1255delA and c.2461A>C. Magnetic resonance imaging (MRI) of the brain showed signal abnormalities in supratentorial white matter (WM), which are conspicuous findings in this disease ${ }^{1}$. Interestingly, MRI also depicted malformations of cortical development - symmetric bilateral parieto-occipital bumpy or pebbly cortical surface (cobblestone malformation) $)^{2}$ (Figure 1).

This report expands $L A M A 2$-related radiological phenotype to include not only WM abnormalities, but also predominantly posterior cerebral cortex changes.

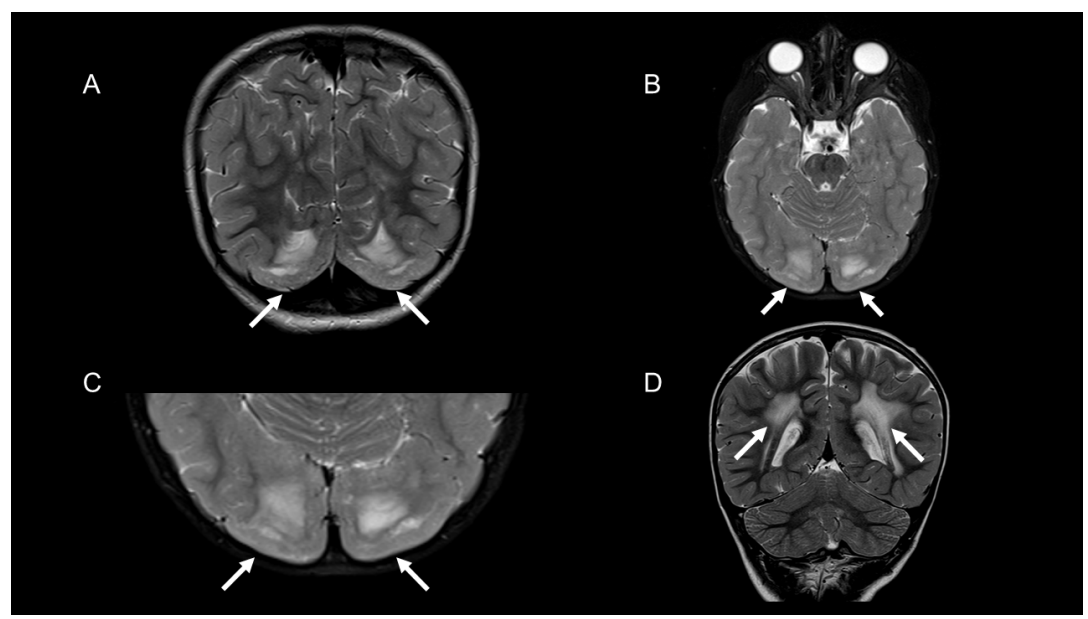

Figure 1. (A) Coronal T2-weighted image (T2WI) showing bilateral and symmetric type II lissencephaly or "cobblestone" lissencephaly (arrows). (B) Axial T2-weighted image (T2WI) showing bilateral and symmetric type II lissencephaly or "cobblestone" lissencephaly (arrows). (C) Axial T2-weighted image (T2WI) with a closer view of the symmetric type II lissencephaly or "cobblestone" lissencephaly (arrows), in contrast with normal cortical development. (D) Coronal T2-weighted image (T2WI) showing signal abnormalities in the periventricular white matter (arrows).

\footnotetext{
'Universidade Estadual de Campinas, Faculdade de Ciências Médicas, Departamento de Radiologia, Campinas SP, Brazil.

${ }^{2}$ Pontifícia Universidade Católica de São Paulo, Faculdade de Ciências Médicas e da Saúde, Departamento de Neuropediatria, Sorocaba SP, Brazil. ${ }^{3}$ Universidade Federal do Rio Grande do Sul, Faculdade de Ciências Médicas, Departamento de Radiologia, Porto Alegre RS, Brazil.

${ }^{4}$ The University of Mississippi Medical Center, School of Medicine, Department of Radiology, Jackson, MS, USA.

5Universidade Estadual de Campinas, Faculdade de Ciências Médicas, Departamento de Neurologia, Campinas SP, Brazil.

LFMB (D) https://orcid.org/0000-0003-2661-4791; FVP (D) https://orcid.org/0000-0002-0828-7806; PMPM (DD https://orcid.org/0000-0001-9041-5047; JAD (iD https://orcid.org/0000-0003-4973-2889; LLV (D) https://orcid.org/0000-0002-9532-1178; TR (iD) https://orcid.org/0000-0001-8453-0313; MCFJ (D) https://orcid.org/0000-0003-0898-2419;FR (i) https://orcid.org/0000-0003-2256-4379

Correspondence: Luiz Fernando Monte Borella; Email: borella.luiz@gmail.com.

Conflict of interest: There is no conflict of interest to declare.

Authors' contributions: LFMB: conceptualization (supporting), data curation (supporting), investigation (supporting), writing-original draft (lead); FVP, PMPM, JAD, LLV, TR, MCFJ: conceptualization (equal), visualization (equal), writing-review \& editing (equal); FR: conceptualization (lead), formal analysis (lead), project administration (lead), visualization (lead), writing-review \& editing (lead).

Received on July 27, 2021; Received in its final form August 11, 2021; Accepted on 19 August 2021.
} 


\section{References}

1. Lamer S, Carlier RY, Pinard JM, Mompoint D, Bagard C, Burdairon E, et al. Congenital muscular dystrophy: use of brain MR imaging findings to predict merosin deficiency. Radiology. 1998 Mar 1;206(3):811-6. https://doi.org/10.1148/radiology.206.3.9494506
2. Jayakody H, Zarei S, Nguyen H, Dalton J, Chen K, Hudgins L, et al. Cobblestone Malformation in LAMA2 Congenital Muscular Dystrophy (MDC1A). J Neuropathol Exp Neurol. 2020 Sep 1;79(9):998-1010. https://doi.org/10.1093/jnen/nlaa062 